\title{
SPACEBORNE BISTATIC SAR SCENE SIMULATION
}

\author{
Rolf Scheiber, Muriel Pinheiro, Marc Rodriguez-Cassola, Pau Prats
}

German Aerospace Center (DLR), Microwaves and Radar Institute, 82234 Oberpfaffenhofen, Germany

\begin{abstract}
Augmenting traditional spaceborne SAR sensors with additional receive-only satellites in close formation enhances the observation space, allowing for single-pass interferomtry in along- and/or across-track with flexible baselines and the potential to build tomographic stacks with reduced temporal decorrelation properties. The feasibility of such add-ons is presently investigated by ESA and other national space agencies and for a variaty of master satellites operating from $\mathrm{X}$ - to L-band. This paper presents a simulation framework for complete scenes dedicated specifically to the analysis of the additional technical requirements imposed by the bistatic SAR imaging geometry with relatively large along-track separation of illuminating master/chief and the receive-only slaves/deputies.
\end{abstract}

Index Terms - bistatic synthetic aperture radar, synchronisation

\section{INTRODUCTION}

Different from interferometric SAR satellite formations with two nearly identical satellites, like the TanDEM-X and Tandem- $\mathrm{L}$ missions, there is also an increased interest in deploying additional receive-only sensors in formation to already existing traditional single sensor SAR missions. These deputy satellites can be much cheaper due to their reduced mass and energy requirements, allowing deployment of several entities within one single launch. ESA considered the deployment of a companion satellite to the Argentinean SAOCOM sensor [1] aimed at enhanced sensing of primarily forested areas, whereas the SESAME mission proposal targeted the measurement of polar ice mass variations and glacier flow by means of two C-band receivers in formation with a Sentinel-1 follow-on mission [2]. In addition, Germany's space agency DLR also considers the additional launch of receive-only satellites for the TerraSAR-X followon mission. For safety reasons, all these mission proposals have in common the relatively large distance of deputies with respect to the illuminating master/chief satellite. This requirement poses several challenges with respect to the SAR

This work has received funding from the European Unions Horizon 2020 research and innovation programme under grant agreement No 687351 (www.s3net-h2020.eu) and from the European Space Agency under ESAESTEC contract 4000117230/16/NL/LvH. image formation algorithm, instrument characterisation and data calibration.

The paper presents a simulation framework dedicated specifically to the analysis of bistatic SAR formations. Section 2 presents the overall architecture of the framework with a brief description of the particularities of the functional blocks related to the bistatic case, whereas section 3 discusses in more detail some of those algorithms, which are either very particular to the chosen large along-track baseline between master and slave, or specially required for a complete scene simulation. These include the bistatic SAR image formation algorithm, it's inversion for reverse processing (raw data generation), as well as the option of synchronisation by one-directional exchange of sync-pulses. Simulation results are used to illustrate the suitability and performance of the selected algorithms. Section 4 concludes the paper.

\section{OVERVIEW OF SIMULATOR ELEMENTS}

The building blocks of the bistatic SAR scene simulator are summarized in the diagram in Fig. 1. The inputs of the simulator are a backscatter map in geographic coordinates together with its corresponding digital elevation model. Further inputs include the radar parameters and the imaging geometry in terms of orbit data of the master satellite. The backscatter map may be of low resolution and derived from a monostatic speckle filtered SAR image acquired using the selected orbit data. This backscatter map is then backgeocoded into the slant-range zero-Doppler geometry of the master acquisition. With respect to the bistatic slave, the following particularities are considered:

- The orbit data are derived from the master orbit in a relative sense using the Clohessy-Wiltshire orbit model and assuming a certain along-track distance. The interferometric across-track baseline changes as a function of latitude.

- For backgeoding the DEM and backscatter information from geographic coordinates into the radar geometry, the beam center geometry of the slave is considered. The azimuth position is determined by the time of the azimuth position of the master (the slave needs to point towards the illuminated area for good SNR) and the 


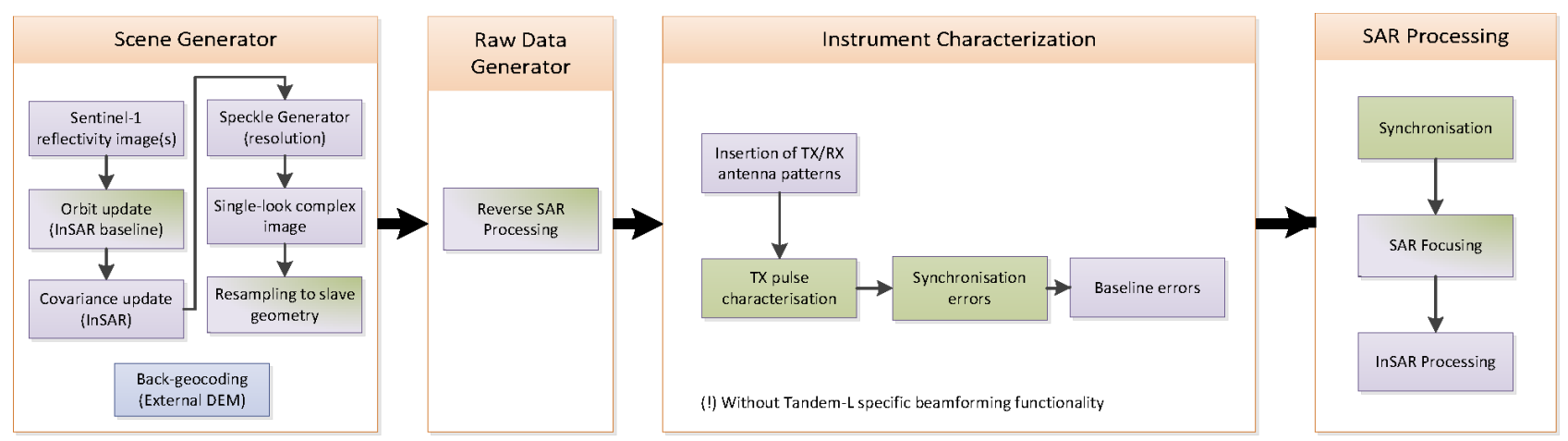

Fig. 1. Elements of the bistatic scene simulator. The functional blocks relevant for the bistatic operation are marked in green.

beam center range position is computed from the master and slave orbit data. Thus, a particular point in the backscatter map will be located at the same azimuth position in the master and slave data sets, but at different ranges.

- The raw data simulation performs first an upsampling according to the SAR sampling parameters followed by the addition of Speckle and noise. The upsampling should allow for a multiple of the PRF to permit the consideration of azimuth antenna side-lobes, influencing the azimuth ambiguity ratio.

- Addition of azimuth antenna patterns: Master and Slave antenna patterns are used to weight the azimuth spectrum. Mis-pointing of the slave antenna can thus be modelled, assuming perfect pointing of the master. Subsampling to regular PRF is done to generate the ambiguities.

- The reverse (and the regular) bistatic SAR processing is performed using as input (output) the beam center geometry imposed by the relatively large along track baseline. This is necessary because of efficiency reasons. It is implemented by a numerical wavenumber domain algorithm [3].

- Synchronisation errors are added to the slave data taking into account the clock parameters. These can be modelled using statistical realisations derived from the spectral characteristics of the oscillator noise, including a constant offset between the center frequencies of the USOs [4]. As an alternative, the measurement of oscillator phase noise from the TanDEM-X mission can be used.

- Bistatic SAR image formation processing starts with a synchronisation step. This can either be based on the evaluation of sync-pulses (see next section) or using the Auto-Sync approach [5].
Ideally, the simulator concludes with the computation of an interferogram between two bistatic slaves with relatively small across- and along-track baseline. Because of the large along-track baseline to the master, the Doppler spectra of master and slaves will be decorrelated.

\section{IMPORTANT ALGORITHMS}

This section provides a more detailed description of two of the main building blocks of the bistatic simulator: the image formation algorithm and its inversion and the synchronisation approach.

\subsection{Bistatic SAR image formation}

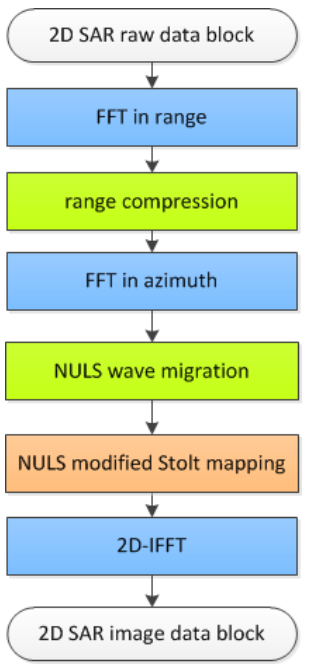

(a)
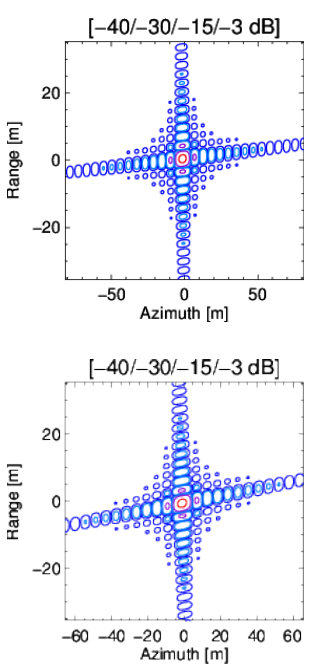

(b)
Fig. 2. Numerical wavenumber domain image formation algorithm [3].(a) Block diagram and (b) focused IRF assuming C-band and 100km (top) and 200km (bottom) along-track baseline between chief and deputy. 
The SAR image formation of the bistatic slaves is performed by a numerical wavenumber domain approach [3]. The method of series reversion is used to compute the $2 \mathrm{D}$ spectrum of the raw data in a numerical least-squre sense, starting from orbit data and the coordinates of a number of points in the scene. The block diagram is given in Fig. 2. The algorithm assumes invariant parameters along azimuth for a complete scene or block of raw data. This assumption was shown to be valid for the SAOCOM-CS L-band case and is applicable also for the C-band SESAME configuration. The inversion of the algorithm has been implemented for the simulation of raw data of extended scenes by simply inverting the sequence of operations. Precautions were required only for the modified Stolt mapping interpolation.

\subsection{Synchronisation approach}

One of the synchronisation approaches implemented considers a cooperative SAR transmitter, able to periodically send sync pulses towards the receive-only deputies. Opposite to the TanDEM-X mission, the receivers do not respond themselves with sync information towards the master. Thus, the precise orbit information is needed to compensate for phase offsets due to the travel time of the sync data. The sync pulses are recorded by the deputies and conditioned to derive range shifts (and corresponding frequency offsets) and phase errors due to slighly different USO frequencies and different phase noise. To do so, the sync pulses are range compressed, their maximum position is evaluated with high precision and used to derive a linear phase ramp in order to properly unwrap the otherwise wrapped phase errors, i.e. the phase of the range compressed peaks (see Fig. 3).

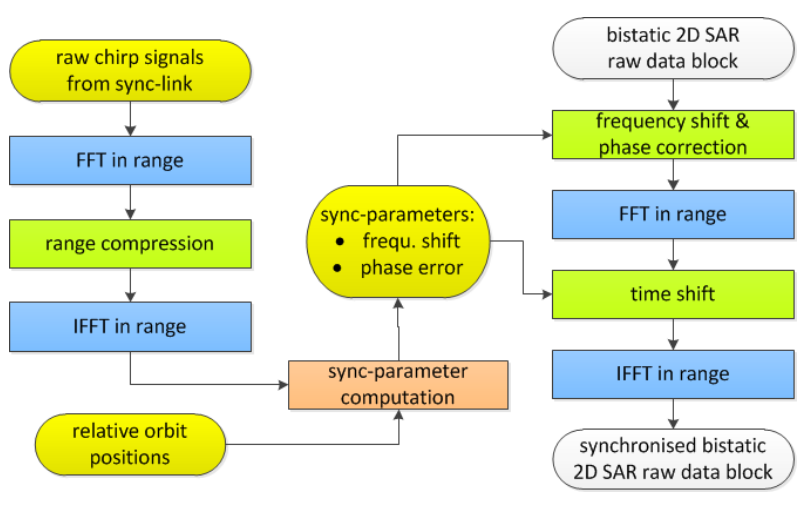

Fig. 3. Synchronisation approach based on evaluation of direct sync signals.

In the simulation, the retrieved sync parameters proved suitable to compensate the differential oscillator phase noise of the bistatic receiver. An example of missing synchronisation is shown in Fig. 4 assuming C-band data with a difference of the $f_{o s c}=10 \mathrm{MHz}$ USO center frequencies of
$\Delta f=4 H z$. The applied phase noise with and without the integrated frequency offset is shown in Fig. 5 (a) and (b), respectively. Without correction the data are characterized by an increasing shift in range up to the order of several $100 \mathrm{~m}$ and defocussing in range and azimuth directions. In this case, the PRFs were assumed synchronized to the PPS signal only at the start of the data take, such that the receive echo window shifts as a function of azimuth time $\left(\Delta r(t)=c_{0} \Delta f / 2 f_{o s c} \cdot t\right.$ [4]).

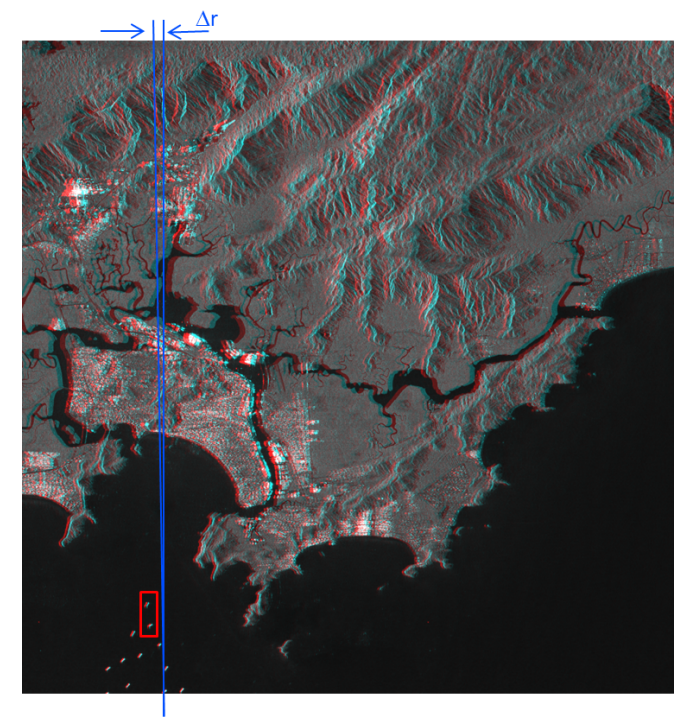

(a)

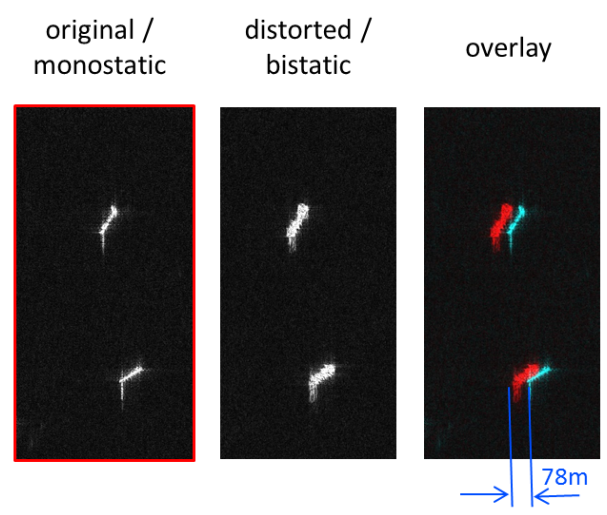

(b)

Fig. 4. Simulation of bistatic synchronisation errors with reference to a monostatic scene in C-band. SAR parameters are according to Sentinel-1 stripmap mode. Oscillator central frequencies differ by $4 \mathrm{~Hz}$. (a) shows the overlay of monostatic and bistatic scenes (extend is $40 \mathrm{kmx} 40 \mathrm{~km}$ ) and (b) presents details on ships waiting to enter the harbor of Santos, Sao Paolo.

A second simulation example is presented in Fig. 6, as- 


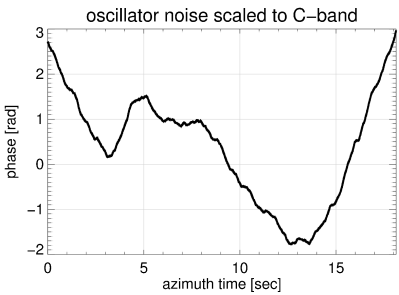

(a)

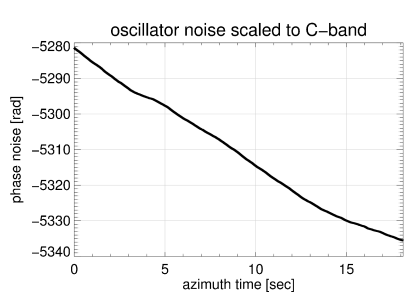

(b)
Fig. 5. Phase noise due to different USO's, scaled to C-band. (a) random realisation with parameters according to [4], (b) same realisation but including a frequency offet of $4 \mathrm{~Hz}$.

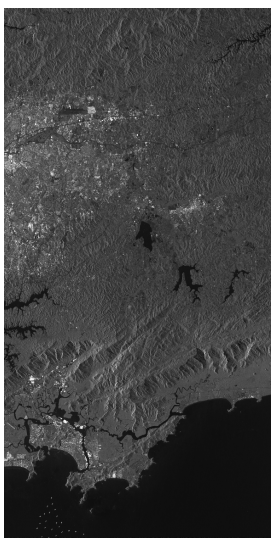

(a)

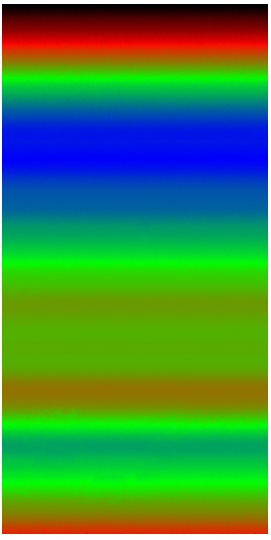

(b)

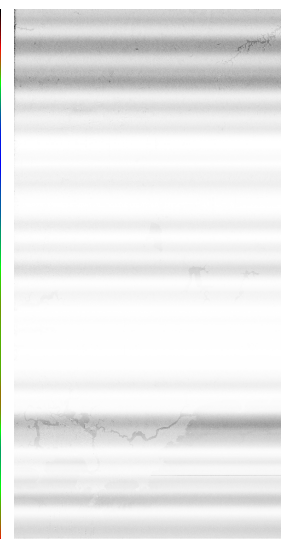

(c)
Fig. 6. Simulation of incomplete bistatic synchronisation: (a) SAR image, (b) interferometric phase error (scaled from $-\pi$ to $+\pi$ ) and (c) coherence (scaled from 0.9 to 1.0)). The effects of the $4 \mathrm{~Hz}$ offset of the receiver oscillator have been compensated, whilst the statistical higher order terms are not corrected. Oscillator noise according to Fig. 5.

suming the same phase error. In this case, the compensation is limited to correcting the effects of the frequency offset $\Delta f$ between transmitter and receiver, i.e. the time shift in range, the frequency shift and the corresponding linear phase correction along azimuth (the situation of known $\Delta f$ ). The higher order phase errors were not compensated. Although in this case the image appears reasonably well focused, the interferometric phase and coherence are strongly affected. (Here the interferograms are formed between the undistorted signal and the distorted one after partial correction.) If complete synchronisation is performed according to Fig. 3, i.e. including time and frequency shifts and phase errors, the residual interferometric phase and coherence errors become negligible (up to a phase constant).

The drawback of this uni-directional synchronisation concept is the need for precise knowledge of relative orbit positions between $\mathrm{Tx}$ and Rx satellites. Especially non-linear varia- tions of the along-track distance need to be known with submillimeter accuracy in order to correctly retrrieve the phase noise. If this is not feasible, bi-directional synchronisation links must be used, as implemented for TanDEM-X. A completely different synchronisation option is the use of AutoSync, which avoids dedicated sync signals and relies instead on the joint evaluation of the recorded bistatic data [5]. In a multi-receiver scenario with large bistatic baselines, a combination of the uni-directional sync approach between Tx and $\mathrm{Rx}$, as presented here, and Auto-Sync between the different $\mathrm{Rx}$-sensors might be a suitable choice and certainly deserves further investigation.

\section{CONCLUSIONS}

We presented a simulation framework for bistatic SAR data. In particular we discussed the additional special building blocks typical for bistatic acquisitions with large along-track baselines between the transmitting and the receiving satellites. In these cases, there is no common Doppler frequency support, such that monostatic and bistatic images are always decorrelated. First simulation results were presented for the image formation algorithm based on $\mathrm{w}-\mathrm{K}$ with numerical least squares kernel (used also for the reverse processing of distributed scenes) and for the simulation and compensation of synchonisation errors.

\section{REFERENCES}

[1] N. Gebert, B. Carnicero Dominguez, M.W.J. Davidson, M. Diaz Martin, and P. Silvestrin, "SAOCOM-CS A passive companion to SAOCOM for single-pass L-band SAR interferometry," in Proceedings of EUSAR. VDE, 2014, pp. 1251-1254.

[2] H. Rott, F. Lopez-Dekker, S Solberg, L. Ulander, T. Nagler, G. Krieger, P. Prats, M. Rodriguez, M. Zonno, and A. Moreira, "SESAME: A single-pass interferometric SEntinel-1 companion SAR mission for monitoring GEO- and biosphere dynamics," in Proceedings of IGARSS. IEEE, 2017, pp. 107-110.

[3] P. Prats-Iraola, M. Rodriguez-Cassola, and A. Moreira, "Investigations on Bistatic SAR Image Formation for the SAOCOM-CS Mission," in Proceedings of EUSAR. VDE, 2016, pp. 1177-1182.

[4] G. Krieger and M. Younis, "Impact of Oscillator Phasse Noise on Bistatic and Multistatic SAR," in IEEE Geoscience and Remote Sensing Letters. IEEE, 2006, vol. 3, pp. 424-428.

[5] M. Rodriguez-Cassola, P. Prats, M. Pinheiro, and et al, "End-to-end Level-0 Data Simulation Tool for Future Spaceborne SAR Missions," in Proceedings of EUSAR. VDE, 2018, pp. 1-6. 